\title{
Article \\ An Analysis Scheme for 3D Visualization of Positron Emitting Radioisotopes Using Positron Emission Mammography System
}

\author{
Md. Rafiqul Islam ${ }^{1,2}\left(D\right.$, Mehrdad Shahmohammadi Beni ${ }^{3,4}$, Shigeki Ito ${ }^{5}$, Shinichi Gotoh ${ }^{6}$, Taiga Yamaya ${ }^{7}$ \\ and Hiroshi Watabe $1,3, *$ (D)
}

1 Graduate School of Biomedical Engineering, Tohoku University, Sendai 980-8579, Japan; islam.md.rafiqul.t5@dc.tohoku.ac.jp

2 Institute of Nuclear Medical Physics, AERE, Bangladesh Atomic Energy Commission, Dhaka 1349, Bangladesh

3 Division of Radiation Protection and Safety Control, CYRIC, Tohoku University, Sendai 980-8578, Japan; mehrdad.shahmohammadi.beni.d5@tohoku.ac.jp

4 Department of Physics, City University of Hong Kong, Tat Chee Avenue, Kowloon Tong, Hong Kong

5 Mirai Imaging Inc., Fukushima 970-1153, Japan; ito@mirai-imaging.com

6 GO Proton Japan Inc., Tokyo 169-0074, Japan; go-proton@outlook.com

7 National Institutes for Quantum Science and Technology, Chiba 263-8555, Japan; yamaya.taiga@qst.go.jp

* Correspondence: watabe@cyric.tohoku.ac.jp; Tel.: +81-22-795-7803

check for

updates

Citation: Islam, M.R.; Beni, M.S.;

Ito, S.; Gotoh, S.; Yamaya, T.;

Watabe, H. An Analysis Scheme for

3D Visualization of Positron Emitting

Radioisotopes Using Positron

Emission Mammography System.

Appl. Sci. 2022, 12, 823. https://

doi.org/10.3390/app12020823

Academic Editor: Chang Ming

Charlie Ma

Received: 17 December 2021

Accepted: 11 January 2022

Published: 14 January 2022

Publisher's Note: MDPI stays neutral with regard to jurisdictional claims in published maps and institutional affiliations.

Copyright: () 2022 by the authors Licensee MDPI, Basel, Switzerland. This article is an open access article distributed under the terms and conditions of the Creative Commons Attribution (CC BY) license (https:// creativecommons.org/licenses/by/ $4.0 /)$.

\begin{abstract}
Proton range monitoring and verification is important to enhance the effectiveness of treatment by ensuring that the correct dose is delivered to the correct location. Upon proton irradiation, different positron emitting radioisotopes are produced by the inelastic nuclear interactions of protons with the target elements. Recently, it was reported that the ${ }^{16} \mathrm{O}(\mathrm{p}, 2 \mathrm{p} 2 \mathrm{n}){ }^{13} \mathrm{~N}$ reaction has a relatively low threshold energy, and it could be potentially used for proton range verification. In the present work, we have proposed an analysis scheme (i.e., algorithm) for the extraction and three-dimensional visualization of positron emitting radioisotopes. The proposed step-by-step analysis scheme was tested using our own experimentally obtained dynamic data from a positron emission mammography (PEM) system (our developed PEMGRAPH system). The experimental irradiation was performed using an azimuthally varying field (AVF) cyclotron with a $80 \mathrm{MeV}$ monoenergetic pencil-like beam. The $3 \mathrm{D}$ visualization showed promising results for proton-induced radioisotope distribution. The proposed scheme and developed tools would be useful for the extraction and 3D visualization of positron emitting radioisotopes and in turn for proton range monitoring and verification.
\end{abstract}

Keywords: proton therapy; positron emitters; PEM; spectral analysis; proton range monitoring

\section{Introduction}

It is well-known that protons have low lateral scattering and therefore deposit energy more locally when compared to photons [1-5]. Hence, the concept of proton range monitoring and verification is important to enhance the effectiveness of proton therapy treatment [6]. The dose deposition of protons increases with depth, and near the end of their range, it reaches its maximum, commonly referred to as the Bragg peak. Considering this, protons have a lower exit dose when compared to photons, which are used in conventional radiation therapy [7-9]. Therefore, protons are highly sensitive to spatial uncertainties [10], which makes range monitoring and verification crucial in therapeutic applications.

Considering proton range monitoring, several strategies have been proposed and investigated, with their own specific advantages and disadvantages [11]. Proton radiography and tomography have both shown promising results for proton range verification, but scattering reduces the resolution of the acquired images [12]. Other direct and costeffective techniques that were reported in previous investigations are the ionoacoustics technique [13] and secondary electron bremsstrahlung (SEB) [14]. In addition, several 
investigators have recently studied Prompt Gamma Imaging (PGI) [15,16] for proton range verification and reported promising results. Another important technique for proton range monitoring and verification is auto-activation Positron Emission Tomography (PET) imaging, which is a non-invasive technique. The proton range is estimated by measuring annihilation photons generated by positron emitting radioisotopes such as ${ }^{15} \mathrm{O},{ }^{11} \mathrm{C}$ and ${ }^{13} \mathrm{~N}$. These positron emitting radioisotopes are generated as a result of nuclear reactions induced by incident protons.

In 1969, Maccabee et al. [17] first proposed the use of the auto-activation PET technique for hadron range monitoring and verification. Later, the proposed auto-activation PET technique was experimentally investigated at a heavy ion therapy facility at the Lawrence Berkeley national laboratory [18]. Furthermore, this technique was found to be promising for monitoring the delivered proton dose in therapeutic applications [19]. There are various PET system configurations that have been used to measure annihilation photons generated by positron emitting radioisotopes in the auto-activation PET technique with the ultimate goal of proton range monitoring and verification [20-24]. For example, full-ring scanners [21,22], which were found to have high detection efficiency but at the same time to incorporate a full ring- ring PET system into beam delivery, which is technically tedious particularly when there are geometric constraints. Non-ring PET systems were developed to circumvent the drawback of full-ring scanners by providing a higher degree of freedom for better patient positioning and installation [25-29]. These non-ring PET systems include the DoPET (Dosimetry-PET) system [26], which is a compact prototype in-beam PET scanner [27], a compact planar PET prototype [28] and a modularized acquisition-based dual-head PET with wider detectors [29]. The main drawbacks associated with these non-ring PET systems are mainly the limited angular coverage and artifacts in the reconstructed images.

Another notable system that has higher spatial resolution and sensitivity compared to previously introduced systems would be the positron emission mammography (PEM) system, which is a dedicated PET system for detecting breast cancer [30]. The PEM system was first proposed by Thompson et al. [31] and inspired the development of several PEM systems such as Clear-PEM [32], composed of a dual-plate detector head composed of LSO (Lutetium oxyorthosilicate) crystals and designed to allow the breast and axilla region field of view (FOV); dual-head planar PEM [33], which has a parallel dual-planar detector geometry composed of LSO crystals and designed for greater flexibility in positioning the object into the FOV; the LBNL-PEM (Lawrence Berkeley National Laboratory-PEM) [34], which has a rectangular geometry, with four detector plates composed of LSO crystals surrounding the breast, and has depth of interaction (DOI) measurements capabilities; the Naviscan PEM flex system [35], which is a specially designed system with two opposite detector heads to fit on a stereotactic mammography unit, with the aim of allowing emission and transmission scans at the same time; the Elmammo (Shimadzu) dbPET (dedicated breast PET) system [36], developed by Shimadzu Co. and designed with three contiguous rings allowing a larger FOV and confirmed high resolution images with four layers of DOI measurement capability; and the Jefferson laboratory PEM system [37], designed to be mounted on a stereotactic biopsy table to perform PEM-guided biopsies.

We have developed a highly sensitive PEMGRAPH (Mirai Imaging Inc., Fukushima, Japan) that consists of a dedicated dual-head PEM system with Pr:LuAG (Praseodymiumdopped Lutetium Aluminum Garnet) crystal and was designed for breast cancer diagnosis [38]. The Pr:LuAG scintillator, with its high light yield, short decay time and good energy resolution, is preferred for angular coverage, while $\mathrm{BaSO}_{4}$ (Barium sulfate) is utilized as a reflector with the crystal to efficiently reflect scintillation photons from Pr:LuAG. PEMGRAPH showed a high spatial resolution of $2.0 \mathrm{~mm}$ (FWHM) in the plane parallel to the detectors [39] and a higher detection sensitivity for breast cancer compared with whole-body PET [40]. Our developed system was found to have higher spatial resolution and higher sensitivity when compared to other commercial PEM devices.

Recently, Cho et al. [41] reported that the ${ }^{16} \mathrm{O}(\mathrm{p}, 2 \mathrm{p} 2 \mathrm{n}){ }^{13} \mathrm{~N}$ reaction has a relatively low threshold energy and it could be potentially used for proton range verification. As a 
result, the ${ }^{13} \mathrm{~N}$ creation may be extracted by computing the gradient between early and late PET scans, and it is found to be highly connected with the Bragg peak. In our recent work, we have employed the Monte Carlo technique to study the generation of the ${ }^{13} \mathrm{~N}$ radioisotope and its potential use to estimate the Bragg peak location in a simulated watergel phantom [42]. In the present work, we have introduced an analysis scheme based on our recent experimentally obtained results from a cyclotron and the PEMGRAPH system for the three-dimensional (3D) visualization of positron emitting radioisotopes (particularly ${ }^{13} \mathrm{~N}$ ). The present analysis scheme uses data obtained from the PEMGRAPH system from a proton irradiated phantom to provide the 3D distribution of positron emitting radioisotopes by using the spectral analysis (SA) approach. All necessary computer programs and raw data used in the present analysis scheme were made open source under the GPLv3 license, which allows users to freely modify, compile and distribute data without any restrictions. To the best of our knowledge, this is the first time such an extensive algorithmic scheme is being proposed, and this will be useful to enhance the effectiveness of proton range monitoring and verification.

\section{Materials and Methods}

\subsection{Experimental Setup and Measurements}

A homogeneous water-gel phantom was prepared by mixing agar powder $\left(\mathrm{C}_{14} \mathrm{H}_{24} \mathrm{O}_{9}\right)$ and water, with a ratio of 1/100 (in gm) (agar powder/water). The container used was high-density polyethylene (HDPE) with dimensions of $8 \times 10 \times 16 \mathrm{~cm}^{3}$ and a wall thickness of $0.15 \mathrm{~cm}$. The phantom geometry and PEMGRAPH are schematically shown in Figure 1. In addition, the material composition of the water-gel and HPDE container are listed in Table 1.
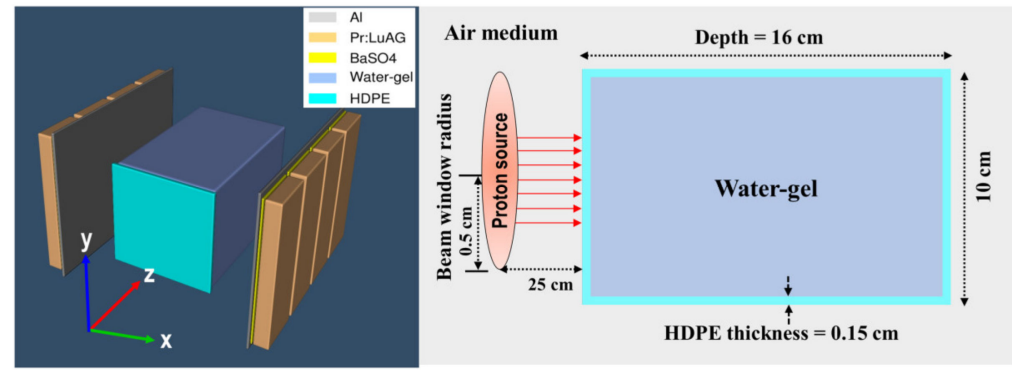

Figure 1. Schematic diagram showing PEM heads and HPDE box containing water-gel being irradiated by a proton beam.

Table 1. Density and material composition (fraction by weight) of HDPE container and water-gel used in the proton irradiation experiment and PEM scan.

\begin{tabular}{ccccc}
\hline Material & Density $\left(\mathbf{g} / \mathrm{cm}^{\mathbf{3}}\right)$ & ${ }^{\mathbf{1}} \mathbf{H}(\mathbf{\%})$ & ${ }^{\mathbf{1 2}} \mathbf{C}(\mathbf{\%})$ & ${ }^{\mathbf{1 6}} \mathbf{O}(\mathbf{\%})$ \\
\hline HDPE box & 0.950 & 14.29 & 85.71 & - \\
\hline Water-gel & 1.010 & 11.00 & 4.650 & 84.35 \\
\hline
\end{tabular}

The phantom was irradiated by a monoenergetic proton beam with $80 \mathrm{MeV}$ energy for $60 \mathrm{~s}$. The proton beam was pencil-like with a beam current of $10.20 \mathrm{nA}$ and a diameter of $\sim 1 \mathrm{~cm}$ produced by an azimuthally varying field (AVF) cyclotron at the Cyclotron and Radioisotopes Centre (CYRIC) facility at Tohoku University, Japan. Considering the current that was used, about $3.8 \times 10^{12}$ protons were used to irradiate the phantom. This value was higher than the typically used clinical beam, which was reported to be around $10^{7}$ to $10^{10}$ protons. We have employed higher number of protons than those used in clinical irradiations mainly to obtain more counts during the PEM scan. It is also well known that proton-induced activity only depends on the target fragmentation. The experimental setup for the irradiation of the water-gel phantom is shown in Figure 2. 


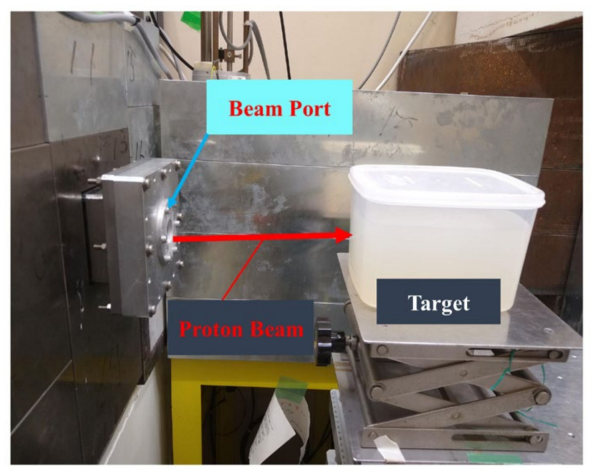

Figure 2. Experimental setup of proton irradiation of water-gel phantom using a pencil-like proton beam with an energy of $80 \mathrm{MeV}$.

The distance between the proton beam port and water-gel phantom was set to $25 \mathrm{~cm}$ (see Figure 2). The proton beam was adjusted to irradiate the center of the water-gel phantom. Upon proton irradiation, different positron emitting radioisotopes are produced by the inelastic nuclear interactions of protons with the target elements. The water-gel was mainly composed of oxygen and carbon (note: hydrogen $\left({ }^{1} \mathrm{H}\right)$ was not considered, mainly because it does not produce stable positron emitting radioisotopes). The list of major nuclear reactions and the positron emitting radioisotopes produced as a result of proton irradiation is shown in Table 2.

Table 2. List of created isotopes, half-life, reaction and threshold reaction energy.

\begin{tabular}{cccc}
\hline Isotope & Half-Life (mins) & Reaction & Threshold (MeV) \\
\hline${ }^{11} \mathrm{C}$ & 20.33 & ${ }^{12} \mathrm{C}(\mathrm{p}, \mathrm{pn}){ }^{11} \mathrm{C}$ & 20.61 \\
\hline${ }^{15} \mathrm{O}$ & 2.037 & ${ }^{16} \mathrm{O}(\mathrm{p}, \mathrm{pn}){ }^{15} \mathrm{O}$ & 16.79 \\
\hline${ }^{13} \mathrm{~N}$ & 9.965 & ${ }^{16} \mathrm{O}(\mathrm{p}, 2 \mathrm{p} 2 \mathrm{n}){ }^{13} \mathrm{~N}$ & 5.660 \\
\hline
\end{tabular}

After proton irradiation, the irradiated water-gel phantom was placed in the PEMGRAPH system; this setup is shown in Figure 3. The irradiated water-gel phantom was scanned for $40 \mathrm{~min}$ with the PEMGRAPH system. The scanning of the irradiated water-gel phantom was started 15 min after the irradiation, mainly due to the time taken to carry the phantom from the irradiation room to the PEMGRAPH room after stopping the proton beam irradiation.

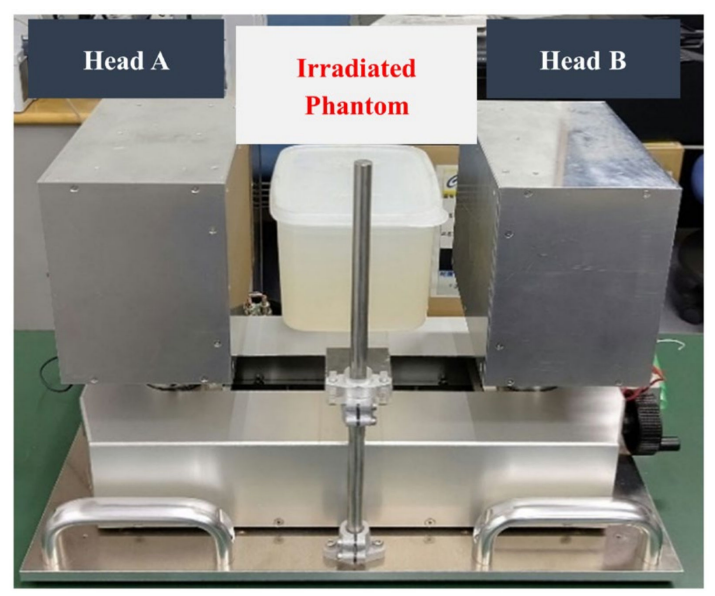

Figure 3. Experimental setup of scanning the irradiated water-gel phantom using a dual-head PEMGRAPH system. 
A prototype PEMGRAPH (Mirai Imaging Inc., Fukushima, Japan) system was employed to scan the irradiated water-gel phantom. The PEMGRAPH system is a highly sensitive and high-resolution open dual-head system [38] that consists of two planar $17.5 \times 21.3 \mathrm{~cm}^{2}$ detector heads, each composed of four modules. There are a total of eight modules implemented in this device. The gap between each module was $0.6 \mathrm{~cm}$. Each module was composed of $20 \times 64$ crystal array of Pr:LuAG with dimensions of $0.21 \times 0.21 \times 1.5 \mathrm{~cm}^{3}$ and was optically isolated by a $\mathrm{BaSO}_{4}$ reflector with a thickness of $0.1 \mathrm{~cm}$. This adds up to a total of $20 \times 64 \times 8=10,240$ Pr:LuAG crystal segments. Each side of the crystal matrix was optically coupled to three units of a $5.2 \times 5.2 \mathrm{~cm}^{2}$ Hamamatsu H8500 position-sensitive photomultiplier tube (Hamamatsu Photonics Co., Ltd., Hamamatsu, Japan). The spatial resolution was $0.2 \mathrm{~cm}$ (at full width at half maximum (FWHM)) in the plane parallel to the detectors [38].

The acquired list mode data were reconstructed to 77 dynamic frames using the 3D iterative maximum likelihood-expectation maximization (MLEM) method [43,44]. The field of view (FOV) of each reconstructed image was $20.35 \times 14.19 \times 14.6 \mathrm{~cm}^{3}$, and the matrix size was $185 \times 129 \times 73$. Therefore, the voxel size of each reconstructed image was $0.11 \times 0.11 \times 0.2 \mathrm{~cm}^{3}$. The experimental data were then analyzed using our previously developed PyBLD software [45] (link: http://www.rim.cyric.tohoku.ac.jp/software/pybld/pybld.html (accessed on: 11 January 2022)). AMIDE (A Medical Image Data Examiner) version 1.0.4 software was used to analyze the images (link: http:/ / amide.sourceforge.net (accessed on: 11 January 2022)) [46]. The one-dimensional (1D), two-dimensional (2D) and three-dimensional (3D) time-course activities for 15, 20, 30 and $55 \mathrm{~min}$ after irradiation were obtained, which provided information on the location and distribution of activity.

\subsection{The Spectral Analysis (SA) Approach}

The spectral analysis approach employs an input function and exponential response function to identify kinetic components of a tracer [47,48]. The SA approach was initially developed for PET kinetics analysis, where it indicates a time-invariant single input/single output model used for the data quantification. During proton therapy, different radioisotopes of positron emitters such as ${ }^{11} \mathrm{C},{ }^{15} \mathrm{O}$ and ${ }^{13} \mathrm{~N}$ are produced by inelastic nuclear interactions of protons with the target elements. Considering that the radioactivity of each radionuclide is one component at time $t$ and the time-activity $A(t)$ of each voxel of the PEMGRAPH image, the concentration $C_{v}(t)$, can be represented as

$$
C_{v}(t)=\sum_{j=1}^{M} A(t) \otimes \alpha_{j} e^{-\beta_{j} t}
$$

where $\otimes$ is the convolution operation and $A(t)$ is the time-activity input function of the system, presumed to be the impulse function in this study. The limit $M$ represents the maximum number of terms to be included in the model and this is, in general, set to a large number, usually 1000 . The values of $\beta_{j}$ are predetermined and fixed in order to cover an appropriate spectral range. For the studies involving short-lived positron emitting isotopes, this range needs to extend to the slowest possible event up to a value appropriate to transient phenomena. Each $A(t) \otimes \exp \left(-\beta_{j} t\right)$ (impulse response function (IRF)) is precalculated. Subsequently, sets of $\alpha_{j}$ values were solved using a non-negative least squares (NNLS) estimator. Once the IRFs are pre-calculated, the estimation sets of $\alpha_{j}$ are entirely linear, and SA can instantly determine groups of $\alpha_{j}$ without iterated computation.

Therefore, SA can be used for large datasets. To extract the short half-life (large $\beta=0.693 / \mathrm{T}_{1 / 2}$ ) ${ }^{15} \mathrm{O}$ radioisotope, a range of $\beta$ was used from 0.005 (athresh in spectral_analysis.py script) to 0.006 (betacut in spectral_analysis.py script) $\mathrm{s}^{-1}$, and for the ${ }^{13} \mathrm{~N}$ radioisotope, a range from 0.001 to $0.002 \mathrm{~s}^{-1}$ was used, respectively. On the other hand, for the longer half-life (small $\beta=0.693 / \mathrm{T}_{1 / 2}$ ) radioisotopes such as ${ }^{11} \mathrm{C}$, the range of $\beta$ from 
0.0005 to $0.0006 \mathrm{~s}^{-1}$ was used. We therefore computed the numerical $S_{v}$ values for each voxel $v$ with the threshold of $\alpha \beta>1.5$ to remove the background region:

$$
S_{v}=\sum_{j=1} \alpha_{j} \beta_{j}
$$

As a result, the SA approach can quickly calculate sets of $\alpha$ and $\beta$ in each voxel. Few positive sets of $\beta$ would be obtained that match the decay constants of the produced radioisotopes.

The SA technique was used to extract ${ }^{11} \mathrm{C},{ }^{15} \mathrm{O}$ and ${ }^{13} \mathrm{~N}$ positron emitting radioisotopes These were extracted separately from the merged images of 77 frames in different regions (see Figure 4). Firstly, the SA technique was applied to 3D merged images that contained 77 frames of $40 \mathrm{~min}$ images (from 15 to $55 \mathrm{~min}$ ). Secondly, the time activity was calculated from the 3D data from 40 frames with $1 \mathrm{~min}$ intervals over $40 \mathrm{~min}$ using four separate regions of interest (ROIs): (1) whole, (2) edge, (3) plateau and (4) Braggpeak region, as shown in Figure 4. Then, the time activity data from 15 to $55 \mathrm{~min}$ (i.e., total 40 frames) were made into a single voxel image for each ROI. Figure 4 shows these four regions, together with their corresponding heights, widths and depths, respectively. The presence of proton-induced radioisotopes for each voxel was then confirmed using the obtained results.

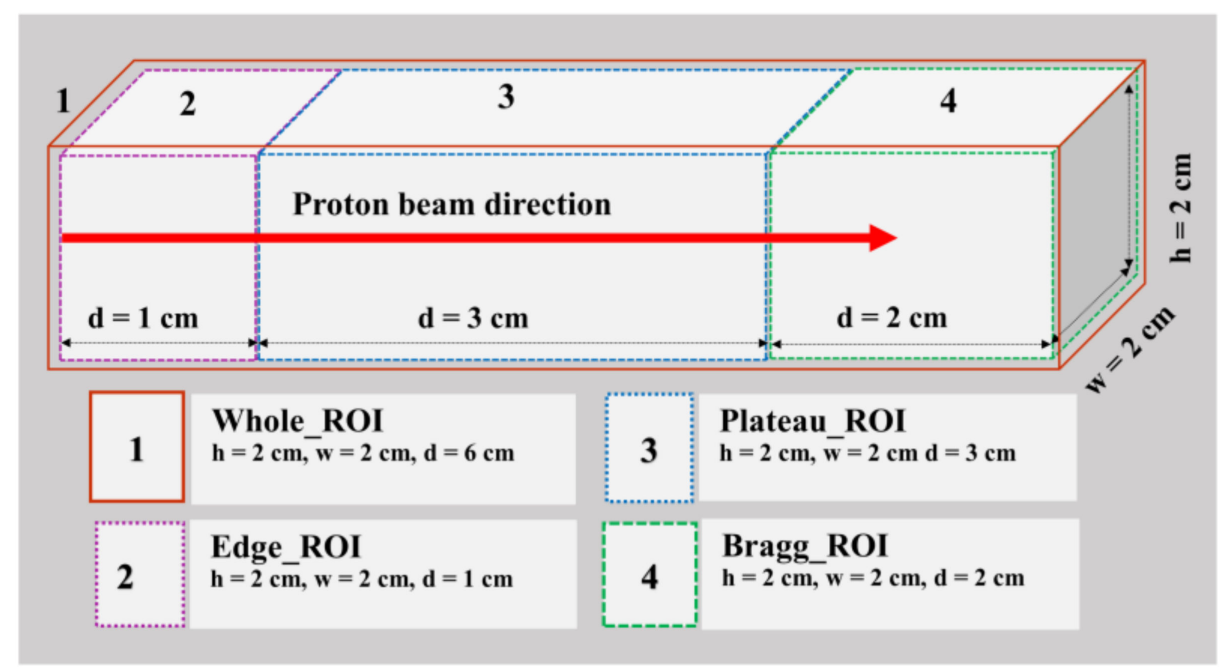

Figure 4. The four regions of interests (ROIs) with their respective height (h), width (w) and depth (d) values used in the spectral analysis approach.

\subsection{Scheme for Extraction and 3D Visualization of Positron Emitting Radioisotopes}

The step-by-step algorithm of the proposed scheme for generating a 3D visualization of positron emitting radioisotopes is shown in Table 3. The algorithm summarizes the main steps for extracting and generating a 3D scatter plot of positron emitting radioisotopes. All the scripts, numerical tools and raw data used in the present scheme can be downloaded as Supplementary material from https:/ / figshare.com/articles/software/An_analysis_scheme_for_3D_ visualization_of_positron_emitting_radioisotopes_using_positron_emission_mammography_ system/17171093 (accessed on: 11 January 2022). 
Table 3. The step-by-step algorithm of the analysis scheme for the extraction and 3D visualization of positron emitting radioisotopes using positron emission mammography.

1. Data acquisition from the PEMGRAPH system (in ascii format in a DAT file).

2. Record the true coincidences as dynamic data from the raw data.

3. Subtract the background data.

4. Sort the data into 77 frames for $40 \mathrm{~min}$.

5. Image reconstruction using the Maximum-Likelihood Expectation-Maximization (MLEM) method (in DICOM format).

6. Convert DICOM data to HDR and IMG format using PYBLD package (using the dicom2ana.py script).

a. Extract the 1D profile data in ascii CSV format (using image.py script).

b. $\quad$ Plot the extracted 1D profile saved into a CSV file.

7. Merge four decay image frames (15, 20,30 and 55 min after irradiation) for visualization of radioisotope distribution with time (using the join_image.py script).

a. $\quad$ Crop the merged images in step 7 (using the crop_img.py script).

b. Visualize the cropped 2D images using the AMIDE software.

8. Generate the 3D scatter plots (using the scatter_3d.py script).

a. The 3D scatter script uses the mplot3d toolkit and the ax.scatter3D() function of the matplotlib library.

9. Perform spectral analysis on the obtained images (using the spectral_analysis.py script).

a. The total of 77 frames of $40 \mathrm{~min}$ image were merged by the join_image.py script and saved as simg_77.img.

b. Crop the merged images in step '9(a)' using the crop_img.py script and saved as simg_77_crop.img.

c. The frame images were prepared in step ' $9(\mathrm{~b})$ ' by frame.py script and saved as simg_77_crop_f.img.

d. The mask images were prepared in step '9(c)' by mk_mask.py and saved as simg_77_crop_fmask.img.

e. Genegate the time information for 77 frames of 40 min dynamic data as time_inf.frame file.

f. Extract the data for positron emitting radioisotopes using python terminal command as:

for ${ }^{11} \mathrm{C}$ radioisotope:

python3 spectral_analysis.py - input $=$ simg_77.img - time $=$ time_inf.frame - output $=$ analysis_c-11 -mask $=$ simg_77-crop_mfsimg.img - betacut $=0.0005$-athresh $=0.0006$ for ${ }^{15} \mathrm{O}$ radioisotope:

python3 spectral_analysis.py - input $=$ simg_77.img - time $=$ time_inf.frame - output $=$ analysis_o-15 - mask $=$ simg_77-crop_mfsimg.img -betacut $=0.005$-athresh $=0.006$ for ${ }^{13} \mathrm{~N}$ radioisotope:

python3 spectral_analysis.py - input $=$ simg_77.img - time $=$ time_inf.frame - output $=$ analysis_n-13 -mask $=$ simg_77-crop_mfsimg.img -betacut $=0.001$-athresh $=0.002$

g. Extract the 1D profile data of analysis output in ascii CSV format (using image.py script).

h. Plot the extracted 1D profile saved into a CSV file.

10. For $1 \mathrm{D}$ spectrum the time activity were calculated as tac.dat from 40 frames with $1 \mathrm{~min}$ interval for four ROIs (1) whole, (2) edge, (3) plateau, and (4) Bragg-peak region (using image_roi.py script).

a. Generate one voxel images of each ROI's time activity in step '10' for example as onev_whole.img (using onev_image.py script).

b. Generate the spectrum of radioisotopes using the python terminal command for a whole ROI as:

python3 spectral_analysis.py - input $=$ onev_whole.img - time $=$ time_inf_roi.frame - output = analysis_out_whole-debug $=1$

11. Plot the concentration $(\alpha)$ versus half-live $(\beta)$ from the terminal output of step ' $10(b)$ '.

12. Generate the $3 \mathrm{D}$ dynamic visualization and movie clips for ${ }^{11} \mathrm{C},{ }^{15} \mathrm{O}$, and ${ }^{13} \mathrm{~N}$ from step ' 9 ' (using scatter_3d.py script). 


\section{Results}

\subsection{PEMGRAPH Measurements}

In order to test the present scheme, we used 77 image frames reconstructed from the obtained 40 min of dynamic data using the PEMGRAPH system. Using the step-by-step scheme presented in Table 3, the 3D, 2D and 1D visualizations are shown in Figure 5. These were reconstructed along the beam direction for 15, 20, 30 and $55 \mathrm{~min}$. In Figure 5, the normalized intensity is displayed along the y-axis. These were normalized with respect to 15 min after irradiation. From the images and 1D profiles shown in Figure 5, we observed two peaks along the beam direction: one at the proton beam entrance and the other near the distal dose Bragg region. In addition, a tail after the peak of the Bragg region was observed. These peaks decayed with time and remained visible for up to $55 \mathrm{~min}$ after the irradiation. Interestingly, the tail almost vanished after $30 \mathrm{~min}$.

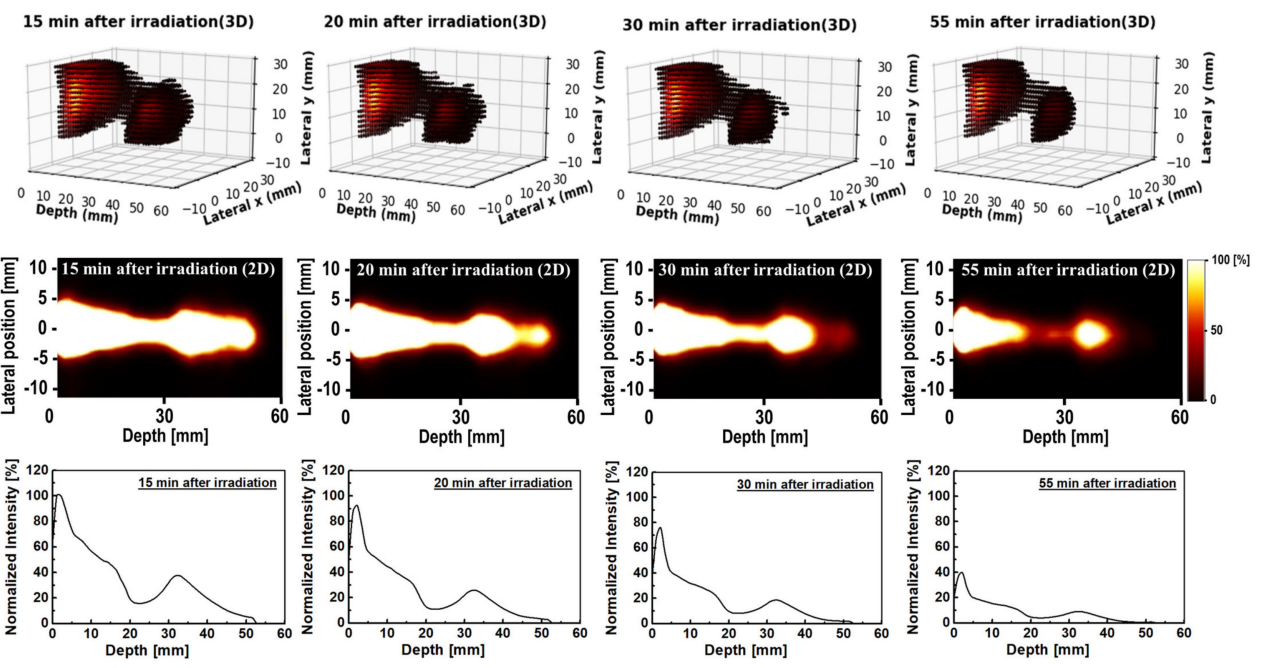

Figure 5. The 3D (first row), 2D (second row) and $1 \mathrm{D}$ (third row) time-course activity covering 15 to 55 min data sets obtained from the PEMGRAPH measurements.

\subsection{Spectral Analysis of PEMGRAPH Measurements}

The spectral analysis (SA) approach was applied to the dynamic time-dependent activity data to quantify different positron emitting radioisotopes. The step-by-step procedure used for this analysis can be found in the proposed scheme shown in Table 3. The 2D images with their associated 1D profiles generated using the SA technique are shown in Figure 6. In Figure 6, the y-axis represents the normalized intensity for each radioisotope. The sum of all radioisotopes (shown as SA_sum in Figure 6), the ${ }^{11} \mathrm{C}$ (shown as SA_ ${ }^{11} \mathrm{C}$ in Figure 6) has two peaks-namely, (1) at the entrance and (2) at a higher depth. On the other hand, the ${ }^{15} \mathrm{O}$ (shown as $\mathrm{SA}_{-}{ }^{15} \mathrm{O}$ in Figure 6) has a peak at a higher depth, and it appeared after the second peak of ${ }^{11} \mathrm{C}$. The ${ }^{13} \mathrm{~N}$ (shown as SA_${ }^{13} \mathrm{~N}$ in Figure 6) peak was found to appear after the ${ }^{15} \mathrm{O}$ peak. In addition, the $1 \mathrm{D}$ profiles are the summation of $2 \mathrm{D}$ images (see Figure 6) along the y-axis (i.e., lateral position); therefore, the shape of 1D profiles would be smooth without showing any sharp cutoffs. 

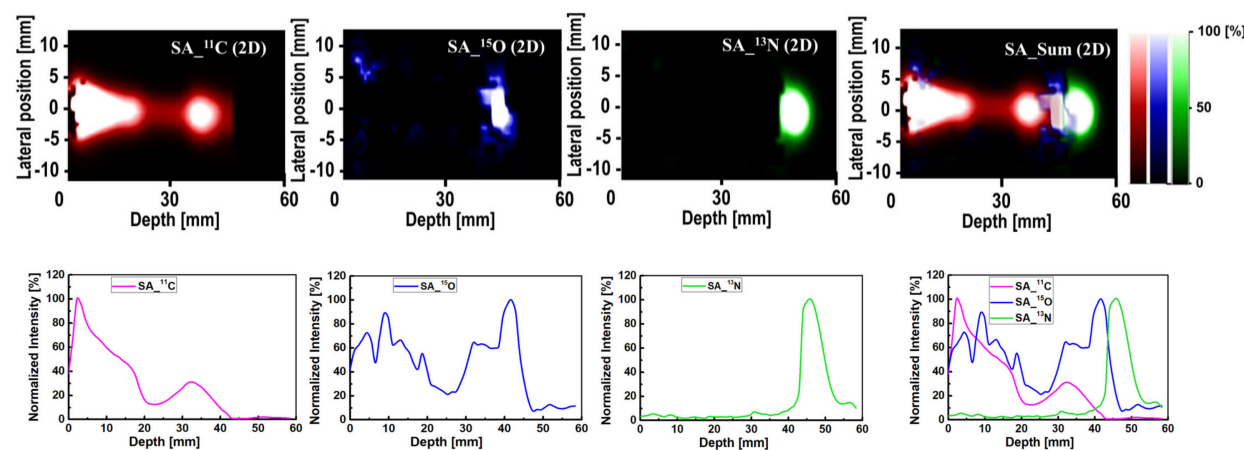

Figure 6. The $2 \mathrm{D}$ (first row) and $1 \mathrm{D}$ (second row) profile of the $\mathrm{SA}$ output ${ }^{11} \mathrm{C},{ }^{15} \mathrm{O}$ and ${ }^{13} \mathrm{~N}$ radioisotopes and their sum (color in 2D plot corresponds to those in 1D profiles).

The ROIs at which the SA method was applied are schematically illustrated in Figure 4 . There are four distinct regions: the whole, edge, plateau and Bragg region (see Figure 4). In Figure 7, the $\mathrm{x}$-axis for all ROIs reflects the extracted half-life of positron emitting radioisotopes $(\log (2) / \beta)$, and the $y$-axis shows the radionuclide concentration, labeled as normalized $\alpha$. Considering Figure 7, we observe that for the whole ROI, the contribution from long-lived radioisotopes was dominant. It is observed that the highest contribution from long-lived radioisotopes is for the edge ROI, which is close to ${ }^{11} \mathrm{C}$ radioisotopes. In the plateau region, it is observed that the total contribution is the same as in the whole and edge regions (i.e., the greatest contribution is from long-lived radioisotopes). On the other hand, for the Bragg ROI, the half-life of radioisotopes was slightly larger than that of the ${ }^{13} \mathrm{~N}$ radioisotopes. From the present analysis scheme, the activity in the distal fall-off region of proton-induced ${ }^{13} \mathrm{~N}$ radioisotopes has been confirmed as the real half-life of ${ }^{13} \mathrm{~N}$ is $\sim 10 \mathrm{~min}$. The discretization in SA and measurement errors (background counts and contributions from multiple radioisotopes) would lead to some differences in the estimated half-life value for a specific radioisotope when compared to its known physical half-life.

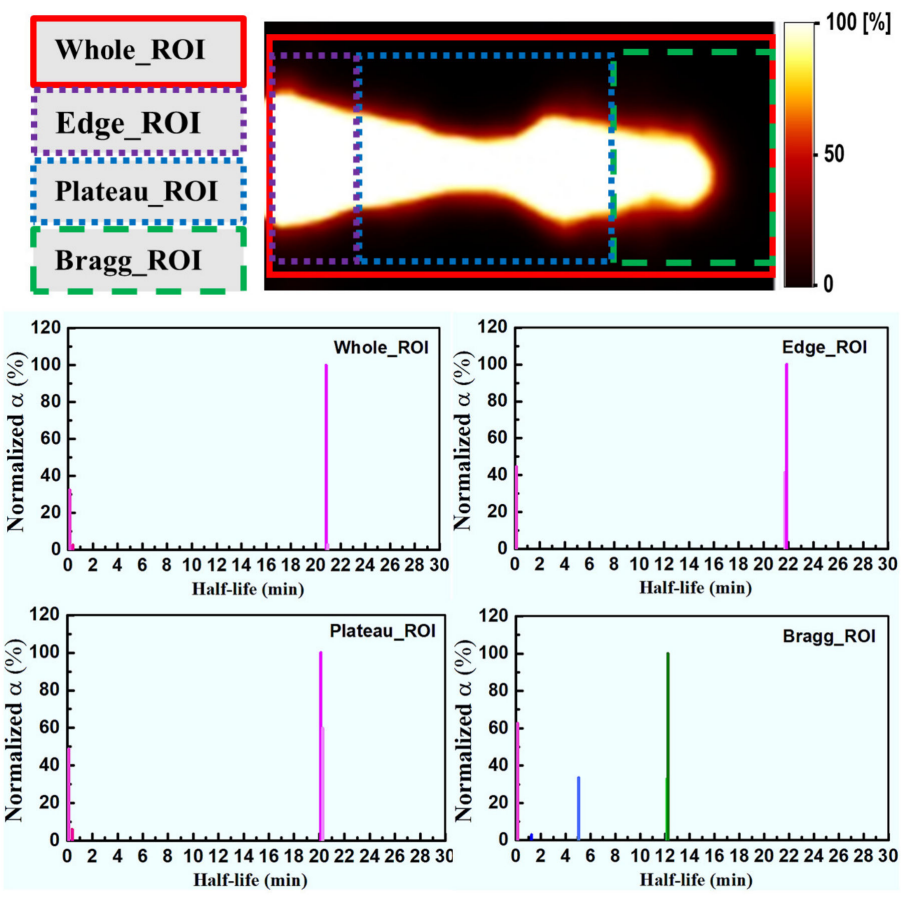

Figure 7. The spectral analysis (SA) for different ROIs. Pink shows no radioisotope components, magenta shows ${ }^{11} \mathrm{C}$, blue shows ${ }^{15} \mathrm{O}$ and green shows ${ }^{13} \mathrm{~N}$ radioisotopes, respectively. 


\subsection{The 3D Visualization of Positron Emitting Radioisotopes}

For a better visualization, the 3D plots of the spatial distribution of extracted radioisotopes were obtained; these were obtained using the analysis scheme shown in Table 3. The generated 3D distributions and $1 \mathrm{D}$ profiles for ${ }^{11} \mathrm{C}$ (shown as SA_${ }_{-}^{11} \mathrm{C}$ ), ${ }^{15} \mathrm{O}$ (shown as $\mathrm{SA}_{-}{ }^{15} \mathrm{O}$ ) and ${ }^{13} \mathrm{~N}$ (shown as $\mathrm{SA}_{-}{ }^{13} \mathrm{~N}$ ) radioisotopes are shown in Figure 8. In addition, the simulated dose distribution for an $80 \mathrm{MeV}$ incident proton beam is also shown in Figure 8 . The step-by-step procedure and required numerical tools for the preparation of 3D distributions are shown in Table 3. The 3D plots generated from the SA images showed the distinct creation of ${ }^{11} \mathrm{C},{ }^{15} \mathrm{O}$ and ${ }^{13} \mathrm{~N}$ peaks, which could be a promising approach for proton range monitoring and verification. From the 1D profiles shown in Figure 8, the ${ }^{11} \mathrm{C}$, ${ }^{15} \mathrm{O}$ and ${ }^{13} \mathrm{~N}$ peaks were found to be at 33, 39 and $46 \mathrm{~mm}$ depths, respectively. Furthermore, the peak of the simulated dose was found to be at $49 \mathrm{~mm}$ depth. Therefore, the offset between the simulated dose and the extracted ${ }^{13} \mathrm{~N}$ peak was found to be $3 \mathrm{~mm}$.
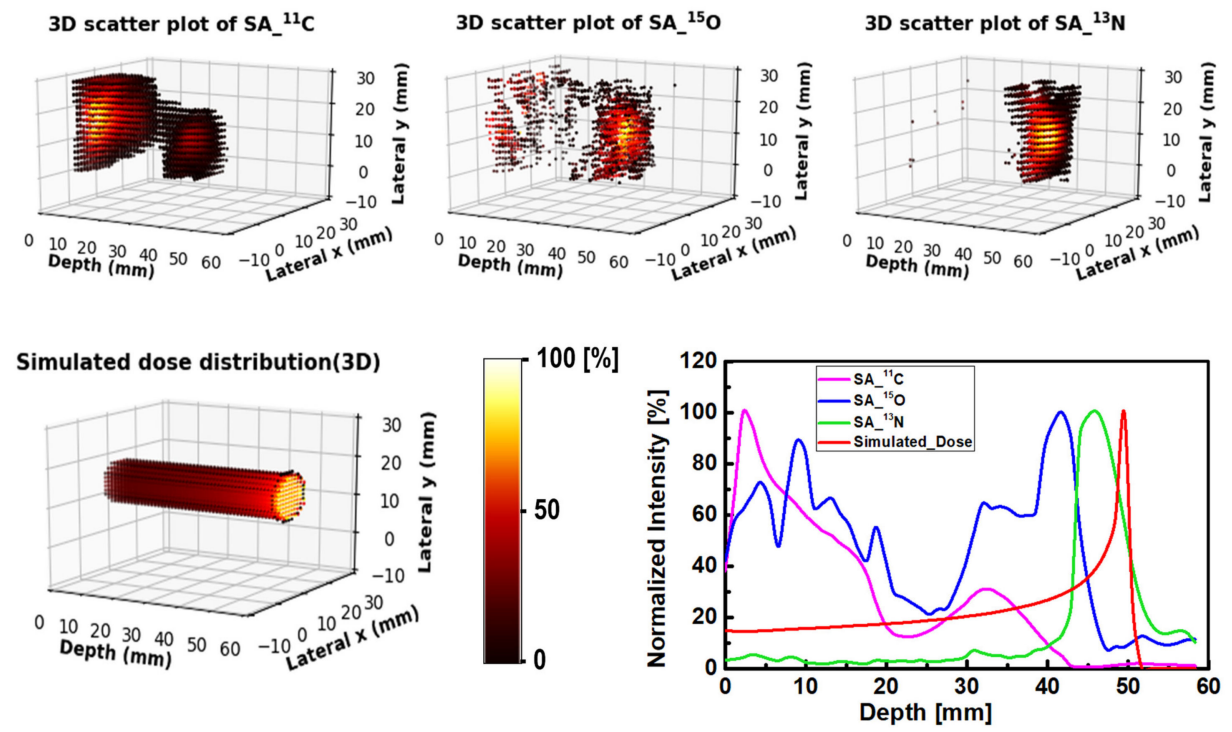

Figure 8. Three-dimensional scatter visualization and the $1 \mathrm{D}$ profiles of ${ }^{11} \mathrm{C},{ }^{15} \mathrm{O}$ and ${ }^{13} \mathrm{~N}$ radioisotopes, along with the simulated dose for the $80 \mathrm{MeV}$ proton beam irradiating water-gel phantom.

\section{Discussion}

Considering the results shown in Figure 5, two peaks can be observed from the PEMGRAPH measurements. In addition to these two peaks, a small tail peak was observed and vanished rather quickly. The main reason for the formation of these peaks was the proton interaction and scattering from the HDPE container used in the proton irradiation experiment; in particular, the wall effect from the HDPE container that mainly contains carbon (note that ${ }^{1} \mathrm{H}$ nuclei do not produce stable positron emitting radioisotopes). Upon the interaction of proton with matter, the protons lose energy, and hence lower energy protons should be found in the deeper parts of the water-gel phantom, and at shallow depths, there will be more higher energy protons; this satisfies the ${ }^{11} \mathrm{C}$ and ${ }^{15} \mathrm{O}$ threshold energies for their production. The threshold energies for the creation of ${ }^{11} \mathrm{C},{ }^{15} \mathrm{O}$ and ${ }^{13} \mathrm{~N}$ were reported to be $20.61,16.79$ and $5.660 \mathrm{MeV}$, respectively [41]. As depth increases and in turn the proton energy decreases, the main production reaction is ${ }^{16} \mathrm{O}(\mathrm{p}, 2 \mathrm{p} 2 \mathrm{n}){ }^{13} \mathrm{~N}$, which has a lower threshold energy compared to other positron emitting radioisotopes. The current finding using the present scheme is in good agreement with previously reported results [41].

The production of proton-induced radioisotopes was confirmed by the SA approach, which was applied to the dynamic data measured using the PEMGRAPH system. In Figure 6, it was confirmed that the two peaks formed at the entrance (i.e., lower depth) and at the end (i.e., higher depth) were mainly due to the ${ }^{11} \mathrm{C}$ radioisotopes. The ${ }^{15} \mathrm{O}$ peak was formed after the second ${ }^{11} \mathrm{C}$ peak, which is also in agreement with the knowledge of the 
threshold energy for the production of these radioisotopes and subsequent energy loss of protons at higher depths. Interestingly, the peak of ${ }^{13} \mathrm{~N}$ radioisotopes was formed after the ${ }^{15} \mathrm{O}$ peak, mainly due to its lower threshold energy of production; this can be seen in the 1D profile plot shown in Figure 6.

Furthermore, the contribution of proton-induced radioisotopes was confirmed in the ROI study. In Figure 7, the main contributing radioisotopes in the entire edge and plateau regions are shown to be ${ }^{11} \mathrm{C}$ radioisotopes. Considering the Bragg region, the ${ }^{13} \mathrm{~N}$ radioisotope makes the highest contribution in the Bragg region. The lowest contribution was found to be from ${ }^{15} \mathrm{O}$ radioisotopes. The SA analysis confirmed the activity in the distal fall-off region of proton-induced ${ }^{13} \mathrm{~N}$ radioisotopes. From the $1 \mathrm{D}$ profiles in Figure 8 , the offset between the simulated dose and extracted ${ }^{13} \mathrm{~N}$ peaks is about $3 \mathrm{~mm}$, which is in good agreement with our recent simulation study [42]. Our reported average offset value between the Bragg and the ${ }^{13} \mathrm{~N}$ peak was found to be about $2 \mathrm{~mm}$ for $80 \mathrm{MeV}$ of incident protons impinging on the water-gel phantom [42]. The present scheme could be applied to inhomogeneous phantoms and small animal experiments after standard irradiation. Further analysis suggests that there is a significant advantage of 3D visualization for proton range verification, especially for inhomogeneous targets (i.e., human organs/tissues).

Considering the generated 3D spatial distribution of positron emitting radioisotopes shown in Figure 8, one can note that the present step-by-step scheme and developed numerical tools can accurately obtain such $3 \mathrm{D}$ visualizations. These $3 \mathrm{D}$ visualizations would be important when investigating non-uniform targets such as organs in the human body or animal subjects. As shown in Figure $8,{ }^{11} \mathrm{C}$ radioisotopes formed two peaks at lower depth (near the entrance) and at higher depth $(\sim 33 \mathrm{~mm})$. The ${ }^{15} \mathrm{O}$ radioisotopes formed a major peak mainly after ${ }^{11} \mathrm{C}$ radioisotopes and before ${ }^{13} \mathrm{~N}$ radioisotopes. The closest peak to the Bragg peak was found to be mainly due to ${ }^{13} \mathrm{~N}$ radioisotopes, thanks to their relatively low threshold energy for production. In order to enhance the 3D visualization, the proposed scheme shown in Table 3 was used to generate movie clips for ${ }^{11} \mathrm{C}$, ${ }^{15} \mathrm{O}$ and ${ }^{13} \mathrm{~N}$ radioisotopes, as shown in Figure 8 . The movie clips can be downloaded from https://figshare.com/articles/software/An_analysis_scheme_for_3D_visualization_ of_positron_emitting_radioisotopes_using_positron_emission_mammography_system/17 171093 (accessed on: 11 January 2022).

\section{Conclusions}

In the present work, a step-by-step analysis scheme using a spectral analysis (SA) approach was introduced. The present scheme determines the 3D spatial distribution of positron emitting radioisotopes, namely ${ }^{11} \mathrm{C},{ }^{15} \mathrm{O}$ and ${ }^{13} \mathrm{~N}$. All the developed numerical tools that are essential for the present scheme have been made open-source and freely available. The present scheme was tested using proton irradiation experiments and measurements from the PEMGRAPH system. The employed water-gel phantom was irradiated with an $80 \mathrm{MeV}$ monoenergetic pencil-like beam. The results obtained from the present scheme showed the formation of a ${ }^{13} \mathrm{~N}$ peak near the Bragg peak, which was found to be in a good agreement with previous investigations. The present scheme and developed tools would be useful for the $3 \mathrm{D}$ visualization of positron emitting radioisotopes and in turn for proton range monitoring and verification.

Supplementary Materials: The movie clips, source codes and raw data can be downloaded at: https: / / figshare.com/articles/software/An_analysis_scheme_for_3D_visualization_of_positron_emitting_ radioisotopes_using_positron_emission_mammography_system/17171093.

Author Contributions: Conceptualization, M.R.I. and H.W.; methodology, M.R.I., H.W. and M.S.B.; software, M.S.B. and H.W.; validation, M.R.I., M.S.B., T.Y. and H.W.; formal analysis, M.R.I., M.S.B., S.I., S.G. and H.W.; investigation, M.R.I., M.S.B. and H.W.; resources, S.I., S.G. and H.W.; data curation, M.R.I., M.S.B. and H.W.; writing—original draft preparation, M.R.I.; writing—review and editing, M.S.B. and H.W.; visualization, M.R.I.; supervision, H.W.; project administration, H.W.; funding acquisition, H.W. All authors have read and agreed to the published version of the manuscript. 
Funding: This research work was funded by Japan Agency for Medical Research and Development (AMED) under Grant Number 20he2202004h0402 and Ministry of Education, Culture, Sports, Science and Technology (MEXT) under the grant number 21F21103.

Institutional Review Board Statement: All involved institutes were informed about the work done.

Informed Consent Statement: Informed consent is not applicable.

Data Availability Statement: https:/ / figshare.com/articles/software/An_analysis_scheme_for_ 3D_visualization_of_positron_emitting_radioisotopes_using_positron_emission_mammography_system/ 17171093 (accessed on: 11 January 2022).

Acknowledgments: The authors thank the Cyclotron and Radioisotopes Center (CYRIC) for the technical support.

Conflicts of Interest: All authors declare that have no conflict of interest.

\section{References}

1. Shahmohammadi Beni, M.; Krstic, D.; Nikezic, D.; Yu, K.N. A Comparative study on dispersed doses during photon and proton radiation therapy in pediatric applications. PLOS ONE 2021, 16, e0248300. [CrossRef] [PubMed]

2. Shahmohammadi Beni, M.; Krstic, D.; Nikezic, D.; Yu, K.N. Medium-thickness-dependent proton dosimetry for radiobiological experiments. Sci. Rep. 2019, 9, 11577. [CrossRef]

3. Shahmohammadi Beni, M.; Ng, C.Y.P.; Krstic, D.; Nikezic, D.; Yu, K.N. Conversion coefficients for determination of dispersed photon dose during radiotherapy: NRUrad input code for MCNP. PLoS ONE 2017, 12, e0174836. [CrossRef] [PubMed]

4. Shahmohammadi Beni, M.; Krstic, D.; Nikezic, D.; Yu, K.N. Realistic dosimetry for studies on biological responses to X-rays and $\gamma$-rays. J. Radiat. Res. 2017, 58, 729-736. [CrossRef]

5. Nikezic, D.; Shahmohammadi Beni, M.; Krstic, D.; Yu, K.N. Characteristics of protons exiting from a polyethylene converter irradiated by neutrons with energies between $1 \mathrm{KeV}$ and $10 \mathrm{MeV}$. PLoS ONE 2016, 11, e0157627. [CrossRef]

6. Knopf, A.C.; Lomax, A. In vivo proton range verification: A review. Phys. Med. Biol. 2013, 58, R131-R160. [CrossRef] [PubMed]

7. Suit, H.; Urie, M. Proton beams in radiation therapy. J. Natl. Cancer Inst. 1992, 84, 155-164. [CrossRef]

8. Shahmohammadi Beni, M.; Krstic, D.; Nikezic, D.; Yu, K.N. Modeling kV X-ray-induced coloration in radiochromic films. Appl. Sci. 2018, 8, 106. [CrossRef]

9. Shahmohammadi Beni, M.; Krstic, D.; Nikezic, D.; Yu, K.N. Modeling coloration of a radiochromic film with molecular dynamicscoupled finite element method. Appl. Sci. 2017, 7, 1031. [CrossRef]

10. Paganetti, H. Range uncertainties in proton therapy and the role of Monte Carlo simulations. Phys. Med. Biol. 2012, 57, R99-R117. [CrossRef]

11. Parodi, K.; Polf, J.C. In vivo range verification in particle therapy. Med. Phys. 2018, 16, e1036-e1050. [CrossRef]

12. Poludniowski, G.; Allinson, N.M.; Evans, P.M. Proton radiography and tomography with application to proton therapy. Br. J. Radiol. 2015, 88, 20150134. [CrossRef]

13. Hayakawa, Y.; Tada, J.; Arai, N.; Hosono, K.; Sato, M.; Wagai, T.; Tsuji, H.; Tsujii, H. Acoustic pulse generated in a patient during treatment by pulsed proton radiation beam. Radiol. Oncol. Investig. 1995, 3, 42-45. [CrossRef]

14. Yamaguchi, M.; Torikai, K.; Kawachi, N.; Shimada, H.; Satoh, T.; Nagao, Y.; Fujimaki, S.; Kokubun, M.; Watanabe, S.; Takahashi, T.; et al. Beam range estimation by measuring bremsstrahlung. Phys. Med. Biol. 2012, 57, 2843-2856. [CrossRef]

15. Min, C.H.; Kim, C.H.; Youn, M.Y.; Kim, J.W. Prompt gamma measurements for locating the dose falloff region in the proton therapy. Appl. Phys. Lett. 2006, 89, 183517. [CrossRef]

16. Pandey, V.K.; Tan, C.M.; Sangwan, V. GaN-based readout circuit system for reliable prompt gamma imaging in proton therapy. Appl. Sci. 2021, 11, 5606. [CrossRef]

17. Maccabee, H.D.; Madhvanath, U.; Raju, M.R. Tissue activation studies with alpha-particle beams. Phys. Med. Biol. 1969, 14, 213-224. [CrossRef]

18. Llacer, J. Positron emission medical measurements with accelerated radioactive ion beams. Nucl. Sci. Appl. 1988, 3, 111-131.

19. Parodi, K.; Enghardt, W. Potential application of PET in quality assurance of proton therapy. Phys. Med. Biol. 2000, 45, N151-N156. [CrossRef] [PubMed]

20. Nishio, T.; Miyatake, A.; Inoue, K.; Gomi-Miyagishi, T.; Kohno, R.; Kameoka, S.; Nakagawa, K.; Ogino, T. Experimental verification of proton beam monitoring in a human body by use of activity image of positron-emitting nuclei generated by nuclear fragmentation reaction. Radiol. Phys. Technol. 2008, 1, 44-54. [CrossRef]

21. Parodi, K.; Ferrari, A.; Sommerer, F.; Paganetti, H. Clinical CT-based calculations of dose and positron emitter distributions in proton therapy using the FLUKA Monte Carlo code. Phys. Med. Biol. 2007, 52, 3369-3387. [CrossRef] [PubMed]

22. Nishio, T.; Miyatake, A.; Ogino, T.; Nakagawa, K.; Saijo, N.; Esumi, H. The development and clinical use of a beam on-line PET system mounted on a rotating gantry port in proton therapy. Int. J. Radiat. Oncol. Biol. Phys. 2010, 76, 277-286. [CrossRef] [PubMed]

23. Yamaya, T.; Inaniwa, T.; Minohara, S.; Yoshida, E.; Inadama, N.; Nishikido, F.; Shibuya, K.; Lam, C.F.; Murayama, H. A proposal of an open PET geometry. Phys. Med. Biol. 2008, 53, 757-773. [CrossRef]

24. Tashima, H.; Yamaya, T.; Yoshida, E.; Kinouchi, S.; Watanabe, M.; Tanaka, E. A single-ring open PET enabling PET imaging during radiotherapy. Phys. Med. Biol. 2012, 57, 4705-4718. [CrossRef] 
25. Enghardt, W.; Debus, J.; Haberer, T.; Hasch, B.G.; Hinz, R.; Jäkel, O.; Krämer, M.; Lauckner, K.; Pawelke, J.; Pönisch, F. Positron emission tomography for quality assurance of cancer therapy with light ion beams. Nucl. Phys. A 1999, 654, 1047c-1050c. [CrossRef]

26. Vecchio, S.; Attanasi, F.; Belcari, N.; Camarda, M.; Cirrone, G.A.P.; Cuttone, G.; Di Rosa, F.; Lanconelli, N.; Moehrs, S.; Rosso, V.; et al. A PET prototype for "in-beam" monitoring of proton therapy. IEEE Trans. Nucl. Sci. 2009, 56, 51-56. [CrossRef]

27. Shao, Y.; Sun, X.; Lou, K.; Zhu, X.R.; Mirkovic, D.; Poenisch, F.; Grosshans, D. In-beam PET imaging for on-line adaptive proton therapy: An initial phantom study. Phys. Med. Biol. 2014, 59, 3373-3388. [CrossRef] [PubMed]

28. Kraan, A.C.; Battistoni, G.; Belcari, N.; Camarlinghi, N.; Cirrone, G.A.P.; Cuttone, G.; Ferretti, S.; Ferrari, A.; Pirrone, G.; Romano, F.; et al. Proton range monitoring with in-beam PET: Monte Carlo activity predictions and comparison with cyclotron data. Phys. Med. 2014, 30, 559-569. [CrossRef] [PubMed]

29. Sportelli, G.; Belcari, N.; Camarlinghi, N.; Cirrone, G.A.P.; Cuttone, G.; Ferretti, S.; Kraan, A.; Ortuño, J.E.; Romano, F.; Santos, A.; et al. First full-beam PET acquisitions in proton therapy with a modular dual-head dedicated system. Phys. Med. Biol. 2014, 59, 43-60. [CrossRef] [PubMed]

30. Hsu, D.F.C.; Freese, D.L.; Levin, C.S. Breast-dedicated radionuclide imaging systems. J. Nucl. Med. 2016, 57, 40S-45S. [CrossRef]

31. Thompson, C.J.; Murthy, K.; Picard, Y.; Weinberg, I.N.; Mako, R. Positron emission mammography (PEM): A promising technique for detecting breast cancer. IEEE Trans. Nucl. Sci. 1995, 42, 1012-1017. [CrossRef]

32. Abreu, M.C.; Aguiar, J.D.; Almeida, F.G.; Almeida, P.; Bento, P.; Carrico, B.; Ferreira, M.; Ferreira, N.C.; Goncalves, F.; Leong, C.; et al. Design and evaluation of the clear-PEM scanner for positron emission mammography. IEEE Trans. Nucl. Sci. 2006, 53, 71-77. [CrossRef]

33. Martinez, J.D.; Toledo, J.; Esteve, R.; Mora, F.J.; Sebastia, A.; Benlloch, J.M.; Fernandez, M.; Gimenez, E.N.; Gimenez, M.; Lerche, C.W.; et al. Fully digital trigger and pre-processing electronics for planar positron emission mammography (PEM) scanners. In Proceedings of the IEEE Symposium Conference Record Nuclear Science, Rome, Italy, 16-22 October 2004; IEEE: Manhattan, NY, USA, 2004; pp. 1778-1781.

34. Huber, J.S.; Choong, W.S.; Wang, J.; Maltz, J.S.; Qi, J.; Mandelli, E.; Moses, W.W. Development of the Lbnl positron emission mammography camera. IEEE Trans. Nucl. Sci. 2003, 50, 1650-1653. [CrossRef]

35. Wollenweber, S.D.; Williams, R.C.; Beylin, D.; Dolinsky, S.; Weinberg, I.N. Investigation of the quantitative capabilities of a positron emission mammography system. In Proceedings of the IEEE Symposium Conference Record Nuclear Science, Rome, Italy, 16-22 October 2004; pp. 2393-2395.

36. Miyake, K.K.; Matsumoto, K.; Inoue, M.; Nakamoto, Y.; Kanao, S.; Oishi, T.; Kawase, S.; Kitamura, K.; Yamakawa, Y.; Akazawa, A.; et al. Performance evaluation of a new dedicated breast PET scanner using NEMA NU4-2008 standards. J. Nucl. Med. 2014, 55, 1198-1203. [CrossRef]

37. Raylman, R.R.; Majewski, S.; Wojcik, R.; Weisenberger, A.G.; Kross, B.; Popov, V.; Schreiman, J.S.; Bishop, H.A. An apparatus for positron emission mammography guided biopsy. In Proceedings of the 1999 IEEE Nuclear Science Symposium, Seattle, WA, USA, 24-30 October 1999; pp. 1323-1327.

38. Yoshikawa, A.; Yanagida, T.; Kamada, K.; Yokota, Y.; Pejchal, J.; Yamaji, A.; Usuki, Y.; Yamamoto, S.; Miyake, M.; Kumagai, K.; et al. Positron emission mammography using Pr: LuAG scintillator-Fusion of optical material study and systems engineering. Opt. Mater. 2010, 32, 1294-1297. [CrossRef]

39. Yanagida, T.; Yoshikawa, A.; Yokota, Y.; Kamada, K.; Usuki, Y.; Yamamoto, S.; Miyake, M.; Baba, M.; Kumagai, K.; Sasaki, K.; et al. Development of Pr: LuAG scintillator array and assembly for positron emission mammography. IEEE Trans. Nucl. Sci. 2010, 57, 1492-1495. [CrossRef]

40. Yanai, A.; Itoh, M.; Hirakawa, H.; Yanai, K.; Tashiro, M.; Harada, R.; Yoshikawa, A.; Yamamoto, S.; Ohuchi, N.; Ishida, T. Newly-developed positron emission mammography (PEM) device for the detection of small breast cancer. Tohoku J. Exp. Med. 2018, 245, 13-19. [CrossRef]

41. Cho, J.; Grogg, K.; Min, C.H.; Zhu, X.; Paganetti, H.; Lee, H.C.; El Fakhri, G. Feasibility study of using fall-off gradients of early and late pet scans for proton range verification. Med. Phys. 2017, 44, 1734-1746. [CrossRef] [PubMed]

42. Rafiqul Islam, M.; Shahmohammadi Beni, M.; Ng, C.Y.; Miyake, M.; Rahman, M.; Ito, S.; Gotoh, S.; Yamaya, T.; Watabe, H. Studies on proton range monitoring by utilizing ${ }^{13} \mathrm{~N}$ peak for proton therapy applications. PLoS ONE 2021, submitted.

43. Shepp, L.A.; Vardi, Y. Maximum likelihood reconstruction for emission tomography. IEEE Trans. Med. Imaging 1982, 1, 113-122. [CrossRef] [PubMed]

44. Shahmohammadi Beni, M.; Krstic, D.; Nikezic, D.; Yu, K.N. Studies on unfolding energy spectra of neutrons using maximumlikelihood expectation-maximization method. Nucl. Sci. Tech. 2019, 30, 134. [CrossRef]

45. Carson, R.E.; Huang, S.C.; Phelps, M.E. BLD: A software system for physiological data handling and model analysis. Proc. Annu. Symp. Comput. Appl. Med. Care 1981, 5, 562-565.

46. Loening, A.M.; Gambhir, S.S. AMIDE: A free software tool for multimodality medical image analysis. Mol. Imaging 2003, 2, 131-137. [CrossRef]

47. Cunningham, V.J.; Jones, T. Spectral analysis of dynamic PET studies. J. Cereb. Blood Flow Metab. 1993, 13, 15-23. [CrossRef] [PubMed]

48. Veronese, M.; Rizzo, G.; Bertoldo, A.; Turkheimer, F.E. Spectral analysis of dynamic PET studies: A review of 20 years of method developments and applications. Comput. Math. Methods Med. 2016, 2016, 7187541. [CrossRef] [PubMed] 